\title{
A Vital Layer of Support: One Safety Net Hospital's Palliative Care Response to the Pandemic
}

\author{
Yijie Xu, MD, Luyi K. Zhang, MD, ${ }^{1,2}$ Robert L. Smeltz, MA, NP, ACHPN, ${ }^{1,2}$ and Susan E. Cohen, MD ${ }^{1,2}$
}

\begin{abstract}
Context: During the coronavirus disease 2019 (COVID-19) pandemic, New York City's public hospitals experienced a significant increase in the number of critically ill patients, especially from minority populations. The palliative care consult service at Bellevue Hospital, therefore, adjusted rapidly to meet the increased needs of our patients and colleagues.

Objectives: To describe the dynamic palliative care needs during a public hospital's COVID-19 surge, including a process to utilize nonpalliative care trained volunteers to meet the increased demand for inpatient palliative care consults.

Confronting the Challenge: Given the flexibility needed during the surge response, the consult team focused on three key elements to meet the system's needs: surge staffing, support, and scale. The consult service expanded into three individual teams to accommodate daily rounds with the medical intensive care and general medicine teams. Nonpalliative care trained community volunteers and internally redeployed providers received targeted training in advanced care planning and were subsequently embedded within the three teams, each led by a palliative care provider. A total of 12 volunteers joined the palliative care team. During eight weeks of the surge, the service cared for a total of 276 patients, 111 of whom were seen by volunteers. Over $50 \%$ of the palliative care patients had limited English proficiency.

Conclusion: The inpatient palliative care consult service structure adapted rapidly in response to the increased need for advanced care planning and support throughout the hospital during the COVID-19 surge. Focusing on three key areas of surge staffing, support, and scale resulted in expert coordination with the hospital and system level leadership, efficient training of volunteer providers, and frequent re-evaluation of response strategies. These elements were vital in allowing the palliative care team to harness the expertise of various volunteer providers to meet the increased demands of a safety net hospital during the COVID-19 pandemic.
\end{abstract}

Keywords: COVID-19; palliative care; pandemic response; safety net hospital

\section{Introduction}

$\mathbf{N}$ EW York City Health and Hospitals (NYC H+H) is the largest public health system in the United States. It is designed as the health care safety net for the hundreds of thousands of NYC residents who are uninsured or underinsured. The patient population is representative of the melting pot of NYC, encompassing a multitude of cultures with $>190$ languages and dialects used. Up to $25 \%$ of patients within the system have limited English proficiency. Bellevue Hospital Center, as the flagship acute care hospital within $\mathrm{H}+\mathrm{H}$ and the oldest public hospital in the United States, has always served some of the most vulnerable patients in the country. Since its founding in 1736, the hospital has cared for patients through various infectious disease outbreaks, including yellow fever, the H1N1 influenza of 1918, the HIV epidemic, Ebola, and, now, coronavirus disease 2019 (COVID-19).

NYC became an epicenter for COVID-19 since its first confirmed case on March 1,2020. There are $>210,000$ cases in NYC with $>54,500$ hospitalizations and 22,365 deaths due to COVID-19 as of June 24, 2020. ${ }^{1}$ The number of cases increased rapidly and the entire NYC health care infrastructure

\footnotetext{
${ }^{1}$ New York University Grossman School of Medicine, New York, New York, USA.

${ }^{2}$ Bellevue Hospital Center, New York, New York, USA.

Accepted January 22, 2021.
} 
endeavored to address the challenges of the pandemic. The inpatient palliative care consult service at Bellevue adapted rapidly to meet the increased need for additional support for our patients and colleagues throughout the hospital. With assistance from leadership at both the hospital and $\mathrm{H}+\mathrm{H}$ system levels, the consult service recruited and trained community providers from various nonpalliative care specialties to implement a strategic response to the COVID-19 pandemic.

\section{Background}

The palliative care consult service at Bellevue Hospital plays an integral role in the care of vulnerable patients with serious illnesses. The team consists of three physicians contributing to 2.3 full-time equivalents (FTEs), one nurse practitioner (one FTE), one chaplain (one FTE), and one social worker (one FTE) to address the palliative care needs of the inpatient population. The service typically sees $\sim 800$ inpatient consultations per year, with an average of 65 consults per month. Our daily inpatient census averages $\sim 30$ patients.

During the COVID-19 pandemic, the total inpatient medicine census increased dramatically with the majority of patients accepted as transfers from other $\mathrm{H}+\mathrm{H}$ hospitals. At the peak of the surge, there were $\sim 400$ medicine patients hospitalized at Bellevue, with 280 on the general medicine service and 99 in the medical intensive care units (ICUs) (Fig. 1). This compares with a typical Bellevue medicine census of 220 patients, with 190 patients on the general medicine services and 40 patients in the medical ICU (Fig. 1). The medicine service swelled from 12 to 22 general inpatient teams and the medical ICU expanded from 1 to 10 teams to accommodate the surging patient volume. Accordingly, the palliative care census grew $350 \%$ during the 8 week peak between April 5 and May 31, which prompted the inpatient palliative care consult service to adapt quickly to accommodate the hospital's unique needs during the pandemic.

The need for additional providers, including palliative care providers, was evident as the number of patients and their acuity increased. NYC government officials announced a statewide call for health care volunteers on March 22, the same day as the start of the New York State PAUSE executive order, when all nonessential businesses closed. ${ }^{2,3}$ A week later, various local and state chapters of the American College of Physicians sent out requests for their members to join volunteer efforts in New York. ${ }^{4}$

\section{Confronting the Challenge}

The palliative care service's census grew from an average of 30 patients to a peak of 105 patients in mid-April (Fig. 2), with a corresponding surge in medical ICU census. In response to the increased number of transfers into Bellevue's medical ICUs starting March 16, two core palliative care providers commenced daily check-ins with the medical ICU team. In the subsequent eight-week surge period, 265 patients died, a $450 \%$ increase compared with the death toll of 59 patients during the same time period in 2019. The palliative care consult census underwent a similar surge between April 5 and May 31 that required ongoing changes to the team structure to meet both patient and hospital system needs. The consult team rapidly implemented changes that targeted the three key concerns detailed hereunder: surge staffing, support, and scale.

\section{Surge staffing}

Early in the pandemic, Bellevue's senior leadership recognized the need for additional staffing to meet the increased needs in the hospital. The NYC H $+\mathrm{H}$ Central Office received numerous offers from volunteers, which included providers already within the $\mathrm{H}+\mathrm{H}$ system, from the local community and those who traveled from other states. A physician volunteer worked closely with the Central Office to coordinate all of the internal redeployment and outside volunteer assignment efforts. ${ }^{5}$ Daily calls between department chiefs and the Central Office started in mid-March and identified needs within the hospital system so volunteers could be deployed to different

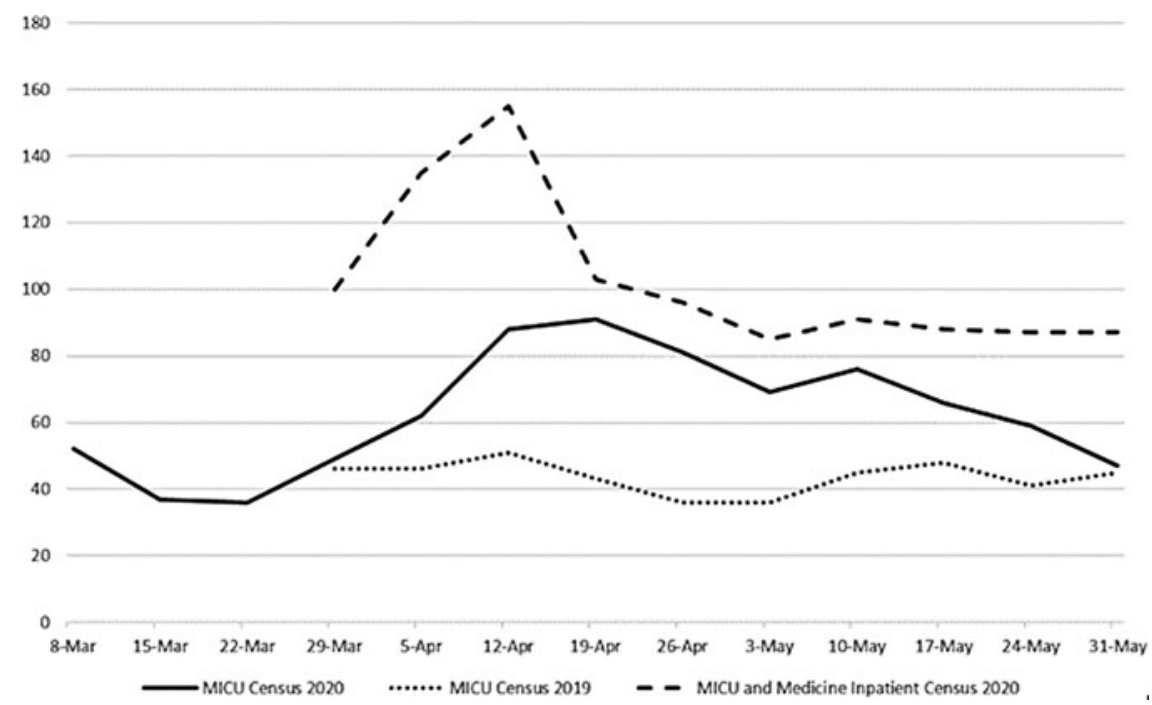

FIG. 1. MICU and medical service (general inpatient medicine and MICU) census during the COVID-19 pandemic, with 2019 MICU census provided for comparison. The surge led to more than a doubling of both MICU (from $\sim 40$ to $\sim 100$ patients) and general medical service (from $\sim 220$ to $>460$ patients) census compared with 2019. COVID-19, coronavirus disease 2019; MICU, medical intensive care unit. 


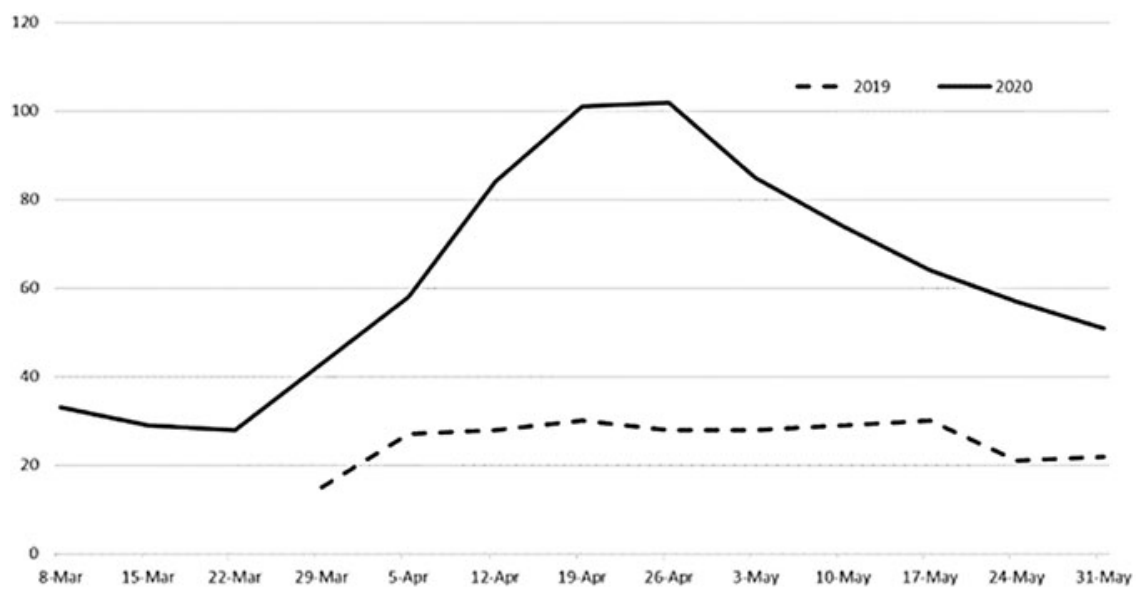

FIG. 2. Palliative care consult service's daily census during COVID-19. The daily census on the consult service increased exponentially from $\sim 30$ patients $(2019$, dotted line) to $>100$ patients (2020, solid line) and remained high throughout the eight weeks of the COVID-19 surge.

departments based on their expertise. Preferred providers for our service included those with palliative care interest or prior training in counseling and conflict resolution. Given the crisis, New York State loosened licensing requirements that allowed Bellevue to expedite the credentialing process from several months to just two days.

Volunteers deployed to the palliative care service spent their first day orienting and shadowing a core palliative care team member (Fig. 3). On the morning of their first day, each volunteer received a manual with essential information that included scripted guides for goals-of-care conversations, updated COVID-19 treatment guidelines, contact information for key collaborators, and logistical information for patients' families (Table 1; see Supplementary Material). Each volunteer began by shadowing a core palliative care provider during an initial consult, most of which were phone calls with family members due to visitor restrictions. By the second day, a volunteer would be able to make calls independently, with a core palliative provider available to provide guidance as needed. From the beginning, the consult service preferentially assigned consults for goals of care and support to volunteer to allow core palli- ative care providers to focus on consults that required more specialized palliative care skills. Therefore, the orientation manual focused on information from VitalTalk's published difficult conversation guides ${ }^{6}$ as well as Bellevue's internal treatment protocols. There was also a video highlighting important tips for documenting in and navigating the electronic medical record. The volunteers, most of whom never received formal palliative care training, were embedded within separate teams, each led by one of the providers from the core palliative care team. Daily rounds discussing all shared patients continued within each individual palliative care team to ensure adequate support for all volunteers and to allow transitions between team members as needed.

Throughout late March to early April, there was an increase in medical ICU census and patient acuity, both of which prompted requests for palliative care consults. The palliative care team quickly realized that the ICU house staff and intensivists were too overwhelmed to request palliative care consults by phone or through the electronic medical record. With the addition of volunteers, the core palliative care team (which had already expanded to include three

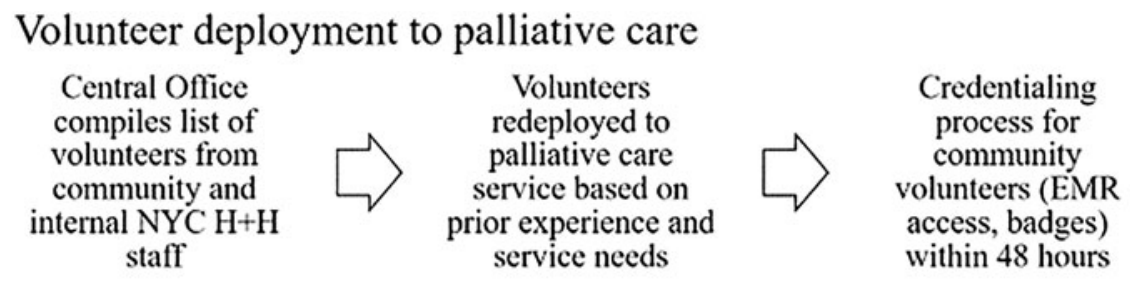

\section{Palliative care volunteer training and onboarding}

Day 1 AM:
Provided orientation
manual containing
logistical
information and
training about
advanced care
planning

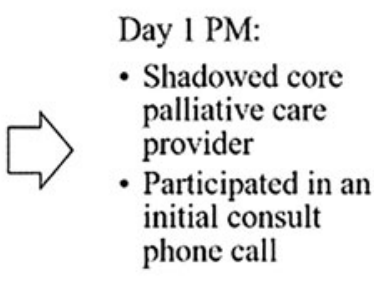

Day 2 and onwards:

- Embedded within palliative care team

- Able to act as independent consultant

FIG. 3. Description of palliative care volunteers' deployment and onboarding process. 
Table 1. Orientation Manual with Essential Information Given to All Volunteers during Onboarding

Palliative care pandemic response manual annotated table of contents

General workflow

General description of daily workflow (with distinction between weekdays and weekends) and timing of rounds with medical ICU and general inpatient medicine teams

Guidelines of important information to share with the primary team during rounds Overall patient trajectory

Current or impending artificial life-sustaining treatments (ventilator, vasopressors, renal replacement therapy, etc.)

Interim goals-of-care discussions

Notifications of death for deceased patients

Palliative care COVID-19

Most recent list of palliative care providers (both core members and volunteers) and their assigned palliative care teams

response teams

Contact information and physical location of all medical teams

Making a phone call

Scripted guide for an initial consult by telephone with family members

Includes examples of useful language for different scenarios, such as routine medical updates, warning shots of difficult news, initiating goals-of-care conversations, and recommendations for next steps based on stated goals

Key contact information

Child life

Patient advocacy

Risk management/ethics

Social work (assigned to all medical ICUs and essential to coordinate videoconferences between patients and families in lieu of in-person visits)

Spiritual care

COVID-19 pandemic adult patient visitor procedure

Information after a death during COVID-19

Most recent information on hospital policy regarding visitors to COVID-19 and non-COVID19 wards

Guidelines about screening procedures, coordination of a visit, what families could expect during the visit, and family visits at the end of the life

Protocol after patient death at Bellevue

Addressed common questions including location of the body, funeral arrangements, virtual services, and contact information for the hospital's Admitting/Decedent Affairs.

Writing notes in epic

Written guide for documentation and a video on navigating the hospital's electronic medical record system.

Appendix

Additional resources for communication (CAPC, VitalTalk) and basic intensive care knowledge (ventilator settings, vasopressors, etc.)

A quick review of COVID-19, common complications, current treatments

The document was also available to all consult team members throughout the surge.

COVID-19, coronavirus disease 2019; ICU, intensive care unit.

physicians, one nurse practitioner, and two palliative care fellows) was further expanded and divided into 2, then 3 distinct teams to accommodate timely daily rounding with the 10 individual medical ICU teams to triage and address the palliative care needs of critically ill COVID-19 patients. Each team of core palliative care and volunteer providers was assigned to cover a corresponding portion of the 10 ICU teams and continued to do so throughout the surge, allowing for enhanced collaboration and continuity of care.

\section{Support}

The consult team saw nearly all patients with multiorgan failure in the ICU and often took over the role of providing routine telephone updates to concerned family members. This offloaded ICU providers who were often at bedside caring for their critically ill patients. Although the palliative care service remained onsite during the surge, a majority of the consults were completed through telephone and later, video conversations with families due to patients' medical acuity and visitor restrictions. The palliative care social worker and chaplain collaborated closely with the hospital's social work and spiritual care departments to use virtual technology to facilitate patient and family communication.

\section{Scale}

The consult service began requesting volunteers from the Central Office at the end of March when the consult census approached $\sim 50$ patients (from a typical baseline of 30) as the concurrently increasing medical ICU census suggested an ongoing demand for palliative care. Staffing needs were based on patient census and estimated at a ratio of five to eight patients for each provider, depending on patient acuity and provider experience or efficiency. As the hospital's needs evolved, the individual palliative care teams expanded from rounding with ICU teams to daily check-ins with inpatient medicine teams to identify patients with high risk of decompensation and to start early advanced care planning with patients themselves.

\section{Assessing the Response \\ Surge staffing}

A total of 12 additional volunteer providers joined the core palliative care consult service from April 6 to May 30. The volunteers included 11 physicians and 1 nurse practitioner. Four providers were available full time for two to four weeks, while the remaining eight were working part time. Four 


\section{Preferred Languages of Patients at Bellevue}

2019

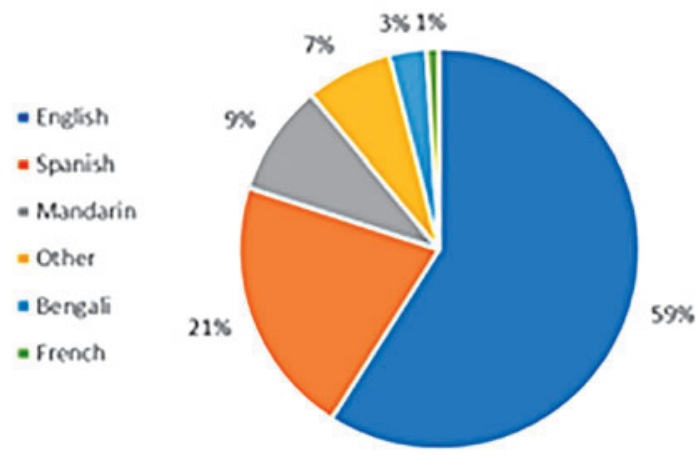

2020

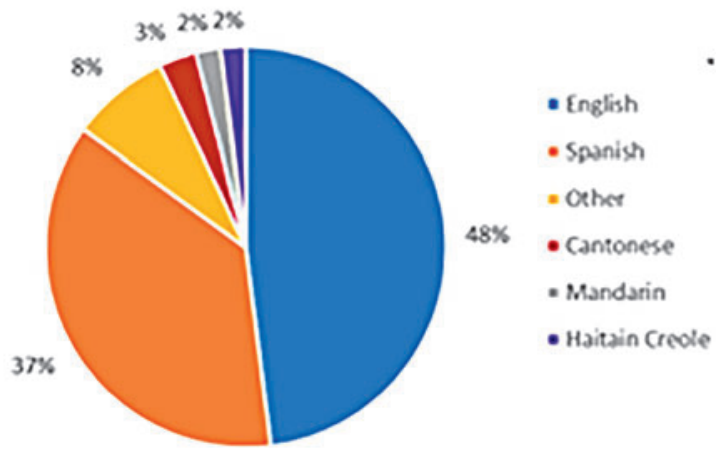

FIG. 4. Bellevue patients' preferred languages at baseline and during COVID-19. There was an increased proportion of non-English-speaking patients at Bellevue during the COVID-19 surge from 40\% (2019) to 50\% (2020), including a significant increase in the percentage of Spanish-speaking patients ( $21 \%$ in 2019 vs. $37 \%$ in 2020).

volunteers were internally redeployed within the $\mathrm{H}+\mathrm{H}$ system, while the remaining eight were community providers. Six of the volunteers were psychiatrists (three from child/ adolescent psychiatry), five from internal medicine, and one from pediatrics. Two volunteers had previous palliative care experience.

During the surge of the pandemic, the number of new daily palliative care consults tripled. The consult daily census increased exponentially from a baseline of $\sim 30$ patients to $>100$ patients during the pandemic (Fig. 2). During the eight weeks of the surge response, the entire palliative care service saw 276 patients. The volunteers saw 117 of these patients and made 663 phone calls. Over $50 \%$ of these patients had limited English proficiency (compared with $40 \%$ at baseline). Spanish, the most commonly preferred foreign language, was spoken by $37 \%$ of the palliative care patients (Fig. 4).

Given the rapid census growth, the palliative care team quickly split into three separate teams to increase efficiency of daily rounds with medical ICU teams and requested volunteers to help with the consult volume. Since a majority of the consults could be completed without direct physical contact with patients with COVID-19, this allowed volunteers with high-risk health conditions to participate in the relief effort. Each team was led by a member of the core palliative care consult service and volunteers were embedded within each team to accommodate the range of consult question. The flexibility with smaller teams allowed the service to respond more easily when general medicine floor teams also requested check-ins with palliative care, as each medicine team was assigned a partner palliative care consult team, similar to the model established in the ICU. Each palliative care team rounded with two to four medical ICU teams and six to eight internal general inpatient teams based on overall census.

Frequent communication was essential during this period of rapid change. Therefore, the entire palliative care team of core and volunteer providers held daily morning calls ( $\sim 10 \mathrm{~min}-$ utes) for general announcements such as COVID-19 patient census, treatment updates, and hospital policy changes. The three separate palliative care teams then split off to conduct their own individual rounds ( $\sim 30-45$ minutes). This ensured familiarity with all patients on a particular team that improved transitions of care when certain team members were unavailable. The newly adapted team structure provided an opportunity to triage consults and assess the appropriateness of existing cases relative to each volunteer's experience. Half of the volunteers were psychiatrists and quickly utilized their own training to support patients and families during the surge. By intentionally assigning the volunteer consults within a specific domain in palliative care (i.e., advanced care planning), the consult service allowed for efficient volunteer orientation and education. The daily team rounds and immediate availability of core palliative care members on site provided ongoing support for volunteers. This system also permitted the core palliative care team to focus on more specialized palliative care consults, such as those with complex family dynamics or advanced symptom management for patients without COVID-19 who continued to be admitted throughout the pandemic.

\section{Support}

The majority of the palliative care consults during COVID-19 were for family support and goals-of-care discussions. Since the majority of patients were intubated and sedated, goals-of-care conversations were conducted with families rather than patients themselves. Although epidemiological studies show that COVID-19 causes more severe disease in older patients or those with chronic illnesses, ${ }^{7}$ many of the seriously ill patients at Bellevue were younger and more independent with limited need for health care before admission. The median age of Bellevue's patients with COVID-19 requiring palliative care was 62 years, compared with a median age of 68 years at other NYC ICUs. ${ }^{8}$ As a result, many patients never had advanced directive discussions with their loved ones. As these patients decompensated, there was an urgent need for palliative care involvement to clarify the goals of care and values.

This need was the greatest in the ICUs, where the massive volume of medically complex and critically ill patients stretched the ICU staff beyond their capabilities. Given the rapidly changing conditions of these patients, the palliative care team expanded our roles for family support and also provided medical updates for the most critically ill ICU patients. The electronic 
medical record did not accurately reflect these changes in real time; therefore, in-person collaboration was essential to provide families with important medical updates. This modification allowed the palliative care team to support family members not only by providing clear and timely medical updates but also by unburdening the primary ICU teams.

Of note, the palliative care team considered seeing patients in the emergency department for early goals-of-care conversations. ${ }^{9}$ However, the majority of the admissions for COVID19 to Bellevue were direct transfers from the other $\mathrm{H}+\mathrm{H}$ hospitals. Bellevue's own emergency medicine department streamlined its workflow to the point that patients only spent a few hours in the closed emergency room. Therefore, we decided to focus our initial efforts and limited resources (personnel and personal protective equipment) by supporting the severely ill patients in the ICUs.

The COVID-19 pandemic in NYC disproportionately affected minority patients. ${ }^{10}$ Non-English-speaking patients usually account for $\sim 40 \%$ of the census on the palliative care consult service. However, during the COVID-19 surge, that number increased to $50 \%$ of all palliative care patients. The percentage of Spanish-speaking only patients nearly doubled from $21 \%$ to $37 \%$ (Fig. 4). As a result, culturally and linguistically appropriate care was essential for these patients. The palliative care team partnered closely with interpreter services to provide clear information to the appropriate family members or surrogate decision makers.

Patients were isolated from their families as a result of visitor restrictions due to hospital and municipal infection control mandates. Goals-of-care conversations were especially challenging by phone given the absence of nonverbal cues, which are an important part of communication in palliative care. The visitor restrictions caused significant distress for some families, some of whom lived in foreign countries and lacked the technology to videoconference with their loved ones. A close partnership with social work and spiritual care eventually allowed families to have daily video calls with hospitalized patients. A palliative care department liaison assisted with orchestrating communication between the Bellevue Command Center and visitor services to allow in-person visits for families of imminently dying patients.

In addition to supporting patients and families, the palliative care team also supported frontline health care providers, many of whom were working outside of their comfort zones under enormous stress. The daily rounds with the ICU and the medical teams allowed the palliative care team to check in with providers during an extremely difficult time. Close collaboration with the primary teams also provided opportunities to teach and model important primary palliative skills. In particular, palliative care demonstrated effective ways of continuing to care for patients and families despite limited available medical interventions to reverse disease courses in critically ill patients. Feedback from ICU and primary medicine teams alike has been overwhelmingly positive with respect to the collegiality and invaluable role palliative care played in Bellevue's strategic response to the COVID-19 pandemic.

\section{Scale}

Owing to the daily rounds with ICU teams, and later the medicine floor teams, the consult service rapidly upscaled its staffing to meet the increased need. As the number of ICU teams increased, the palliative consult service split into two, then three individual teams to allow closer collaboration with the individual ICU providers. Palliative care was in close communication with the $\mathrm{H}+\mathrm{H}$ Central Office to request additional volunteers based on the consult need based on previously mentioned ratios of five to eight patients per provider. The team added new weekend in-house staffing in early April given the high patient acuity and volume, which allowed the team to work with volunteers who needed more flexible schedules. Full-time providers who worked one or both weekend days were given a corresponding weekday off to maintain two days off within a seven-day period.

Likewise, as the census decreased in early May, the team ceased weekend in-house staffing and released our volunteers. The service condensed to two teams for two weeks, and returned to one main service once the census reached $\sim 30$ patients by May 20 .

\section{Future Implications}

Bellevue is a large urban hospital with a high proportion of underserved and linguistically diverse patients. This population may not be representative of those seen at other institutions. Furthermore, we relied significantly on volunteer providers, whose onboarding and training may be more difficult to arrange with a smaller or nonexistent core palliative care consult teams. This reliance upon volunteers across the hospital also meant providers with varying levels of primary palliative skills cared for COVID-19 patients. The palliative care team had to navigate this unfamiliarity by educating colleagues in addition to orienting new palliative care volunteers.

The immediacy and urgency of our interventions required that necessitated swift implementation and execution of various response strategies, which did not allow for a more structured project that could have had more directly measurable outcomes. Our experience during the COVID-19 response suggests the importance of palliative care services in a large-scale pandemic. This article describes a process for a palliative care service to expand rapidly and adjust workflow to meet the unique needs of a safety net hospital during the COVID-19 pandemic. The existing palliative care consult service utilized the expertise of a wide range of volunteers, many of whom lacked formal palliative care training, to meet the increased patient and provider needs during the COVID19 surge. Through these efforts, the team increased awareness of primary palliative care and provided essential support for patients, families, and colleagues. We hope this model can help other institutions develop similar ways of recruiting volunteers or redeploying existing staff to improve palliative care access and support in a pandemic or disaster setting.

\section{Acknowledgments}

We thank Dr Ann Ostrovsky for organizing the volunteer efforts at Bellevue. We also thank Dr Ian Fagan, Ms. Karen Hewitt, and Dr Amit Uppal for their contributions to data collection and analysis.

\section{Authors' Contributions}

All authors contributed equally to article conception and design. R.S. contributed to data acquisition. R.S. and Y.X. 
contributed to data analysis and interpretation. All authors contributed to article drafting, revision, and approval for final submission.

\section{Funding Information}

No funding was received for this article.

\section{Author Disclosure Statement}

No competing financial interests exist.

\section{Supplementary Material}

Supplementary Data

\section{References}

1. NYC Health. COVID-19: Data. https://www1.nyc.gov/site/ doh/covid/covid-19-data.page. 2020. (Last accessed June 25, 2020).

2. New York State. Amid ongoing COVID-10 pandemic, Governor Cuomo announces 40,000 health professionals have signed up to volunteer as part of the state's surge healthcare force. https://www.governor.ny.gov/news/amidongoing-covid-19-pandemic-governor-cuomo-announces40000-health-professionals-have-signed. 2020. (Last accessed May 25, 2020).

3. New York State. New York State on PAUSE. https:// coronavirus.health.ny.gov/new-york-state-pause. 2020. (Last accessed May 25, 2020).

4. American College of Physicians. New York chapter's urgent call for assistance. https://www.acponline.org/about-acp/ chapters-regions/united-states/maryland-chapter/meetingsand-events/new-york-chapters-urgent-call-for-assistance. (Last accessed May 25, 2020).

5. Keeley C, Long TG, Cineas N, et al.: Staffing for the surge: Expanding the New York City public hospital workforce during the COVID-19 pandemic. Health Aff 2020;39: 1426-1430.

6. VitalTalk. COVID resources. https://www.vitaltalk.org/ covid-resources/. (Last accessed November 13, 2020).

7. Perrotta F, Corbi G, Mazzeo G, et al.: COVID-19 and the elderly: Insights into pathogenesis and clinical decisionmaking. Aging Clin Exp Res 2020;32:1599-1608.

8. Richardson S, Hirsch JS, Narasimhan M, et al.: Hospitalized with COVID-19 in the New York City area. JAMA 2020;23:2052-2059.

9. Lee J, Abrukin L, Flores S, et al.: Early interventions of palliative care in the emergency department during the COVID19 pandemic. JAMA Intern Med 2020;180:1252-1254.

10. NYC Health. COVID-19: Rates of cases, hospitalizations, and deaths by race/ethnicity group. https://www1.nyc.gov/ assets/doh/downloads/pdf/imm/covid-19-deaths-race-ethnicity05142020-1.pdf. 2020. (Last accessed May 21, 2020).

Address correspondence to: Yijie $\mathrm{Xu}, \mathrm{MD}$

New York University Grossman School of Medicine New York, NY

USA

E-mail: yijie.xu@nyulangone.org 